\title{
AREA AND REPRESENTATION OF SURFACES
}

\section{LAMBERTO CESARI}

What is surface and what is area? This question is not a trivial one, because study of the literature reveals that there are several relevant concepts of surface and area, just as there are several relevant concepts of curve and length (see [1, I.I, II.5, V.4]; numbers in square brackets refer to the bibliography at the end of this paper). These concepts are not only related to one another, but they are also related to the solution of many problems in which they play an essential part. My purpose is to discuss the connection of such concepts with calculus of variations and to show how the slow development of some of them is closely related to advances in calculus of variations.

During the war years I succeeded in developing a complete theory for Fréchet surfaces and Lebesgue area, which was related to previous work of McShane and Radó. It was with great pleasure that I learned after the war that, at the same time and independently, Rado and other mathematicians in this country had developed a theory founded upon the same fundamental ideas. The results obtained are partly overlapping and partly complementary. This allows us to combine our results into a single theory. Now this parallel development is not due to chance, but rather to an underlying common aim that has been a constant guide in our respective efforts: the aim to obtain a more general basis for the theory of double integrals in parametric form in calculus of variations. Indeed, the theory for such integrals has not yet been finally settled, as has been noticed by both Radó and Tonelli.

The concepts of curve and surface, length and area, were presented at other meetings of this society by J. W. T. Youngs [59] from a general point of view. Therefore I will limit myself here to showing only how much our concepts owe to calculus of variations, and then I will discuss the most recent and important results concerning the problem of area and representation of surfaces.

A complete list of all papers which are related to this topic would be too extensive for the purposes of the present paper. The bibliography at the end is not meant to be complete in any sense. The recent book of Radó, Length and area [1], will be used as a general refer-

An address delivered before the Annual Meeting of the Society in Columbus, Ohio, on December 29,1948, by invitation of the Committee to Select Hour Speakers for Summer and Annual Meetings; received by the editors February 21, 1949. 
ence. Several papers that have been published since that book appeared are included in the bibliography, provided that they are directly related to our main topic.

The first question which led to the modern theory of calculus of variations was the problem of Dirichlet, that is, the problem of determining a function $f(x, y)$ whose values are given on the boundary of a Jordan region $A$ and for which the integral

$$
\iint_{A}\left[\left(\frac{\partial f}{\partial x}\right)^{2}+\left(\frac{\partial f}{\partial y}\right)^{2}\right] d x d y
$$

assumes its minimum value. From the fact that the set of the values assumed by such an integral has a finite lower bound, mathematicians inferred the existence of the minimum. This became a general principle, called the principle of Dirichlet, and it gave rise immediately to numerous applications. The most general of these was certainly the one made by Riemann, who based his theory of abelian functions entirely on this principle. After criticism by Weierstrass the principle of Dirichlet was abandoned and the question of determining a harmonic function that assumed assigned values on the boundary of a Jordan region was settled in another way by Neumann, Schwarz, and Poincaré.

The possibility of giving a direct demonstration of the principle of Dirichlet was recognized by Arzelà, who was the first to try to prove the principle of Dirichlet in connection with concepts of the functional analysis of Volterra. Arzelà did not succeed in this, but his research, continued by Hilbert, led the latter in 1900 to the first proof of the principle. This achievement gave rise to a good deal of research between 1900 and 1904. We mention the work of Lebesgue, Bolza, Carathéodory, who gave existence theorems for problems of calculus of variations under hypotheses which were still rather restrictive. However, the method created by Hilbert did not yield further results, so that several authors later turned to other procedures for the solution of maximum and minimum problems in calculus of variations. Of these authors I would like to mention Hadamard and Levi.

The reason for this fact was realized later when Tonelli observed first that the concept of semicontinuity introduced in 1906 by Baire in the theory of real functions should be introduced as the fundamental concept. Tonelli noted that the most common functionals of a curve in calculus of variations are lower or upper semicontinuous. In particular the "length" of a curve is the simplest example of a functional of a curve that is lower semicontinuous. Indeed all the regular 
positive functionals of a curve are lower semicontinuous.

Arising from the functional analysis of Vito Volterra and the notion of semicontinuity introduced by Tonelli into calculus of variations, the direct method of calculus of variations for functionals of a curve was developed for the last thirty years and, with Menger, we may say that the results obtained are now classical. This work was carried on by mathematicians all over the world. I mention only Graves, McShane, Reid in the United States and Tonelli, Baiada, Cinquini, Manià in Italy.

A new step is due to Bouligand and Menger. The former observed that, upon introducing a suitable metric in the space of curves, each integral along a curve, which is positive, regular, and therefore lower semicontinuous, can be considered as a "generalized length" for the curve. The latter observed that by means of this approach, combined with the Weierstrass integral, it is possible to extend the direct method to functionals of a curve in any abstract space. With this modern extension the ordinary length of a curve becomes only the most important "functional of comparison," but these functionals are always lower semicontinuous.

I would like to mention, however, that the concept of lower semicontinuity, which also had a fundamental role in Marston Morse's topological theory of calculus of variations, has been replaced in his more recent work by the concept of upper reducibility. Also in this connection operational concepts and metrization seem to have increasingly wide application.

Let us now turn our attention from curves to surfaces, from line integrals to surface integrals, from length to area.

Of all definitions of surface area which were known at the beginning of this century there was only one which was completely general and which satisfied the principle of lower semicontinuity: the one proposed by Lebesgue in his memoire in 1900 [33]. Geöcze worked a long time on this definition during the period from 1905 to 1916. Tonelli [53] used it in 1915 for a demonstration of the minimum property of the sphere known as the isoperimetric inequality. This inequality was proved recently by Radó [44] for general closed continuous surfaces. As proved by Radó, the fundamental isoperimetric inequality states that

$$
V^{2}(S) \leqq L^{3}(S) /(36 \pi),
$$

where $L(S)$ is the Lebesgue area of the closed surface $S$ and $V(S)$ is the volume enclosed by $S$ according to the following definition. Let $i(x, y, z)$ be the "topological index" of the point $(x, y, z)$ with respect 
to $S$, where $i(x, y, z)=0$ for points on $S$. Then $V(S)$ is defined as the integral of $|i(x, y, z)|$. Just as the Lebesgue area $L(S)$ is a lower semicontinuous area, so also is the volume $V(S)$ considered by Radó a lower semicontinuous volume.

Of course, if the surface $S$ is an ordinary surface or, at least, admits of a lipschitzian representation, then all definitions of area and of volume agree and the above inequality is to be expected. But with the concept of Lebesgue area and the preceding definition of volume, this inequality is completely general and holds for general surfaces even in cases in which not all definitions of area and volume agree.

Let us recall the main concepts of the theory of curves

$$
C: \quad x=x(t), \quad y=y(t), \quad z=z(t), \quad a \leqq t \leqq b .
$$

A curve $C$ has finite length $l(C)$ if and only if the functions that represent it are of bounded variations; the length of a curve is always greater than or equal to the value of the classical integral:

$$
l(C) \geqq \int_{a}^{b}\left(x^{\prime 2}+y^{\prime 2}+z^{\prime 2}\right)^{1 / 2} d t ;
$$

the length of a curve is equal to the classical integral if and only if the functions that represent the curve are absolutely continuous.

These three fundamental facts have been extended by Tonelli [54] to the Lebesgue area of nonparametric surfaces

$$
z=f(x, y), \quad(x, y) \in A .
$$

Tonelli introduced appropriate definitions for bounded variation and absolute continuity of the function $f(x, y)$. The main results are the following: the surface $S$ has finite Lebesgue area if and only if the function $f(x, y)$ is of bounded variation; the Lebesgue area $L(S)$ of $S$ is always greater than or equal to the value of the classical integral:

$$
L(S) \geqq \iint_{A}\left(1+p^{2}+q^{2}\right)^{1 / 2}, \quad p=\frac{\partial f}{\partial x}, \quad q=\frac{\partial f}{\partial y} ;
$$

the Lebesgue area of the surface $S$ is equal to the classical integral if and only if the function $f(x, y)$ is absolutely continuous [54].

It is also remarkable that these concepts of bounded variation and absolute continuity introduced by Tonelli for the problem of Lebesgue area for nonparametric surfaces $S$ have had, from 1926 to the present, extensive and varied applications in the theory of differential equations, in potential theory, and in the theory of double and multiple Fourier series. But one of the main applications is cer- 
tainly that in calculus of variations to double integrals over a nonparametric surface $S[56]$ :

$$
I_{S}=\iint_{A} f(x, y, z, p, q) d x d y .
$$

A complete theory based on the concept of semicontinuity has been created since 1929 by Tonelli, Cimmino, Cinquini, Scorza in Italy and by Graves, Bliss, McShane, Morse, Reid in the United States. In this theory the problem of Dirichlet and also the more difficult problem of Plateau were settled by Courant, Douglas, McShane, Morse, Radó, and Tonelli [55]. At the same time some more general variational problems for surfaces in parametric form

$$
S: \quad x=x(u, v), \quad y=y(u, v), \quad z=z(u, v), \quad(u, v) \in A,
$$

were studied by McShane [34, 35, 36, 37] and Radó [45].

From the above discussion of applications in calculus of variations it appears advisable to choose for general surfaces a concept of area which satisfies the fundamental principle of semicontinuity, namely the Lebesgue concept of area, which was so useful for nonparametric surfaces.

Naturally for applications in which the principle of semicontinuity is not so important, and even for calculus of variations if we do not base it on the concept of semicontinuity, other definitions of area can also be considered. In this connection I mention the definitions of Minkowski and Carathéodory. Carathéodory introduced a general and convenient concept of linear measure or length, and of superficial measure or area, of any point set in space. A complete theory for rectifiable sets is due to A. S. Besicovitch. This theory was extended by A. P. Morse and J. F. Randolph by use of a more general concept of Carathéodory measure; it was further extended to point sets of finite area by Besicovitch, Busemann, Federer, and other authors. This theory has been very carefully worked out and is related to recent trends in the theory of integration. Another definition of area is due to Banach [4] and Vitali [57]; the theory for this area was carried out by Banach and Schauder.

Of course all definitions of area coincide in ordinary cases, and they also coincide when the surface has a lipschitzian representation; in the general case they no longer coincide.

Today we have various definitions of area which satisfy the principle of semicontinuity, but in all such cases it follows from the research of Rado and myself that they coincide with the Lebesgue area. First of all let us mention the so-called Geöcze area $G(S)$ in the 
sense used by McShane [34] and Morrey [41], which is introduced by means of the topological index of a point with respect to a closed plane curve. I have shown [16] that the lower semicontinuous Geöcze area $G(S)$ coincides with the Lebesgue area $L(S)$. Radó $[1, \mathrm{~V} .1]$ has introduced a "lower area" that is also lower semicontinuous. I have completed Radó's proof that it coincides with the Lebesgue area $[1, \mathrm{~V}, 4.8]$. Quite recently Radó modified previous definitions of Cauchy and Favard in order to obtain two new definitions of area satisfying the principle of semicontinuity. Jointly with Helsel [46] and Mickle [47], he has shown that these two concepts of area also coincide with the Lebesgue area.

In 1928, in his communication at the International Mathematics Meeting of Bologna, Radó [48] had already stated the conjecture that each lower semicontinuous functional over a surface which coincides with the elementary area for polyhedral surfaces must coincide with the Lebesgue area for any surface, at least under very weak and natural conditions. From this point of view, which we shall call the tendency for an axiomatic definition of area, contributions have been made by Kempisty, Fréchet, Scorza, Zwirner, Stampacchia.

On the same occasion Rado [48] also observed that it would be desirable to define axiomatically the area $\alpha(S)$ of the surface $S$ by means of a Dedekind cut in the field of real numbers. Indeed, if we require $\alpha(S)$ to be a lower semicontinuous functional coinciding with the elementary area upon polyhedral surfaces, then it is easy to prove that $\alpha(S) \leqq L(S)$. Therefore $L(S)$ is an upper bound for the area $\alpha(S)$. If we require $\alpha(S)$ to be greater than or equal to the value of the Geöcze area $G(S)$, which is intuitively the smallest possible interior area, then $\alpha(S)$ also has a lower bound

$$
G(S) \leqq \alpha(S) \leqq L(S) .
$$

Radó's conjecture that $G(S)=L(S)$ has now been proved by myself [16], and therefore the Lebesgue area is, in this sense, determined by an intuitively motivated Dedekind cut.

The notion of surface $S$ that we use is not the set of points covered by it, but comes from the consideration of all possible "transformations" or "representations"

$$
T: \quad x=x(u, v), \quad y=y(u, v), \quad z=z(u, v), \quad(u, v) \in A,
$$

that are equivalent in the Fréchet sense. Each of these equivalent transformations gives a representation of the surface $S$. It is well known that the Lebesgue area $L(S)$ does not depend upon the repre- 
sentation, but is a property of the surface itself. If we require the notion of Fréchet equivalence to conserve the orientation of the boundary $A^{*}$ of the Jordan domain $A$, as McShane did [34], then we speak of "oriented Fréchet surfaces"; otherwise, simply of "Fréchet surfaces" or "surfaces."

Important research in set topology, related to this concept of a surface, was carried out by Kerékjárto, R. L. Moore, Whyburn, Wiener, and Wilder. Of this research I point out the theory of upper semicontinuous collections and the theory of cyclic elements; these led to the topological notions of the cactoid of R. L. Moore and of the hemicactoid of Morrey. A complete revision of the applications of this theory to surface area theory has been made by Radó and his school, so that we now have a very simple and clear theory of the topology of Fréchet surfaces [1, II]. Each transformation $T$ is the product of a monotone transformation $M$ and of a light transformation $L: T=L M$. This is a monotone-light factorization of the transformation $T$. $M$ maps $A$ onto a Peano space $\mathfrak{M}$ that is called the "middle space"; $L$ maps $\mathfrak{M}$ onto $S$. For two different representations $T_{1}$ and $T_{2}$ of the same surface $S$, we have

$$
T_{1}=L M_{1}, \quad T_{2}=L M_{2} ;
$$

that is, we have different monotone factors but the same light factor $L$. The middle space is the cactoid or hemicactoid of the previous theory. Youngs has succeeded in giving a complete intrinsic topological characterization of the notion of Fréchet equivalence. These results of Youngs are now in the process of publication [62].

If we divide $A$ into a finite number of Jordan domains $A_{1}, A_{2}, \cdots$, $A_{n}$ by curves $\gamma_{1}, \gamma_{2}, \cdots, \gamma_{m}$ and let $S_{1}, S_{2}, \cdots, S_{n}$ be the continuous surfaces defined by (1) upon $A_{1}, A_{2}, \cdots, A_{n}$ respectively, then we have

$$
L(S) \geqq \sum_{i=1}^{n} L\left(S_{i}\right)
$$

It is of the greatest importance to have conditions sufficient for equality to hold in this relation. Theorems of this type, or additivity theorems, have been proved and applied by Radó [1] and Youngs [59].

In 1924, in order to extend to surfaces the theory of Jordan and Tonelli for length of curves, Banach [4] and Vitali [57] gave, independently of each other, a theory for the area of surfaces in parametric form: 


$$
S: \quad x=x(u, v), \quad y=y(u, v), \quad z=z(u, v), \quad(u, v) \in A .
$$

They were the first to observe that properties of a surface, for instance that of having finite area, depend upon properties, not of the single functions $x, y, z$ of the representation, but of pairs of these functions:

$$
T_{1}:\left\{\begin{array}{l}
y=y(u, v), \\
z=z(u, v),
\end{array} \quad T_{2}:\left\{\begin{array}{l}
z=z(u, v), \\
x=x(u, v),
\end{array} \quad T_{3}:\left\{\begin{array}{l}
x=x(u, v), \\
y=y(u, v) ;
\end{array}\right.\right.\right.
$$

that is, they depend upon properties of the three "flat surfaces" or the plane transformations which are the three projections of the surface upon the three coordinate planes. From this point of view the results obtained by Banach and Vitali constitute a formal extension of the theory of arc length, in the sense of Jordan and Tonelli, to general continuous surfaces given in parametric form. However, they made use of a concept of surface area which differs from Lebesgue area and lacks the property of lower semicontinuity.

In quite different ways, and independently, Radó [1, IV.4, IV.5] and I $[8,12,14]$ introduced concepts of plane transformations of bounded variation. These concepts, however, are completely equivalent, as Radó [50] has shown.

Furthermore, during the war I [8] proved the following

THEOREM 1. The Lebesgue area of a general surface $S$ is finite if and only if the three plane transformations $T_{1}, T_{2}, T_{3}$, which are obtained by projecting the surface upon the three coordinate planes, are all of bounded variation.

This result can also be expressed by saying that the Lebesgue area of the surface $S$ is finite if and only if these three projections $T_{1}, T_{2}, T_{3}$, considered as flat surfaces, all have finite Lebesgue area.

One result in Radó's theory, as well as in mine, is that each continuous surface of finite Lebesgue area has generalized Jacobians $J_{1}(u, v), J_{2}(u, v), J_{3}(u, v)$ almost everywhere in $A$. For such surfaces these generalized Jacobians are $L$-integrable functions in $A$, generalize the ordinary Jacobians

$$
\frac{\partial(y, z)}{\partial(u, v)}, \quad \frac{\partial(z, x)}{\partial(u, v)}, \quad \frac{\partial(x, y)}{\partial(u, v)}
$$

formed with ordinary first partial derivatives, and coincide with them provided the ordinary Jacobians exist almost everywhere.

Radó, Reichelderfer, and I, at the same time and independently, have each proved the following

Theorem 2. If the surface S has finite Lebesgue area, then the plane 
transformations $T_{1}, T_{2}, T_{3}$ are of bounded variation and have generalized Jacobians $J_{1}(u, v), J_{2}(u, v), J_{3}(u, v)$ a.e. in $A$; furthermore the Lebesgue area is greater than or equal to the classical integral

$$
L(S) \geqq \iint_{A}\left(J_{1}^{2}(u, v)+J_{2}^{2}(u, v)+J_{3}^{2}(u, v)\right)^{1 / 2} d u d v .
$$

This result is also in agreement with the classical theory of Banach concerning functions of a set.

In completely different ways Radó and I have each introduced concepts of absolutely continuous plane transformations. These concepts are also equivalent, as Radó [50] has shown. Radó and I have each proved the following

THEOREM 3. The Lebesgue area is equal to the classical integral if and only if the three plane transformations $T_{1}, T_{2}, T_{3}$, projections of the surface upon the coordinate planes, are absolutely continuous.

I $[11,25]$ have also proved several tangential properties for the most general surfaces of finite Lebesgue area.

The classical results of Jordan and Tonelli for curves and the results of Tonelli for surfaces in nonparametric form are now completely extended by these theorems concerning Lebesgue area to surfaces in parametric form. These theorems also establish the fact that the concepts of total variation and absolute continuity for plane transformations which were introduced by Radó, Reichelderfer, and myself are completely adequate.

It is of interest to note that the concept of plane transformation of bounded variation and the concept of absolutely continuous plane transformation have been applied to the important problem of the transformation of areas or of double integrals by Radó, Reichelderfer, Helsel, and Mickle here in the United States, by myself [24] in Italy, obtaining equivalent and very general formulas of transformation.

The Kolmogoroff principle has been proved and generalized by Mickle and Helsel [32] and has been applied to the stretching process for surfaces and in the proofs of additivity theorems. The main results that $\mathrm{I}$ have mentioned solve the fundamental problems for the Lebesgue area of general surfaces. The following is another very important problem.

We know that each continuous rectifiable curve has at least one representation for which the length is equal to the classical integral and such a representation is obtained in a very simple way by choosing as parameter the length of the arc from a fixed point to a variable 
point of the curve. We also know that for non-rectifiable curves the problem of a special representation has been solved by Marston Morse.

For surfaces we have the following two problems, which may be termed representation problems:

(i) Given a surface $S$ of finite Lebesgue area, does there exist a representation for which the Lebesgue area is given by the classical integral calculated with generalized Jacobians?

(ii) Given a surface $S$ of finite Lebesgue area, does there exist a representation for which the functions $x, y, z$ have first partial derivatives a.e. in $A$ and for which the Lebesgue area is given by the classical integral calculated with ordinary Jacobians?

McShane studied these problems because of important applications to the calculus of variations and, in particular, to the Plateau problem. McShane [36] showed that such a special representation exists for the so-called "saddle surfaces." More generally, Morrey [43 ] found such a representation for the surfaces that we call "open nondegenerate," that is, according to the nomenclature of Radó, those for which the middle space is a 2-cell. For such surfaces McShane and Morrey found a new type of representation, the so-called "almost conformal representation." A representation (1) of a surface $S$ is called "almost conformal" if

(a) the functions $x, y, z$ are absolutely continuous in the sense of Tonelli in $A$;

(b) the first partial derivatives $x_{u}, x_{v}, \cdots, z_{v}$ are $L^{2}$-integrable functions in $A$;

(c) we have $E=G, F=0$, a.e. in $A$, where $E=x_{u}^{2}+y_{u}^{2}+z_{u}^{2}, G=x_{\text {, }}^{2}$ $+y_{v}^{2}+z_{v}^{2}, F=x_{u} x_{v}+y_{u} y_{v}+z_{u} z_{v}$.

For such representations the Lebesgue area is necessarily equal to the classical integral. The theorem of Morrey can now be rephrased as follows: Each open nondegenerate surface of finite Lebesgue area admits of at least one almost conformal representation upon $A$ for which the area is given by the classical integral.

We know that a Jordan domain can be represented upon a circle in a conformal way. We also have the Schwarz theorem: each polyhedral surface which is open nondegenerate can be represented upon a circle in a conformal way; that is, it admits of a representation (1) for which properties (a) and (b) hold and also for which the relations $E=G, F=0$ hold everywhere in $A$ with the exception of a finite set of points, the points which correspond to the vertices of the polyhedral surface. By an almost conformal representation of any open nondegenerate surface we mean one for which the exceptional points 
form a set of measure zero. The theorem of Morrey thus appears as a generalization of Schwarz's theorem.

The almost conformal representations of an open nondegenerate surface of finite Lebesgue area satisfy a minimum principle $[15,17]$ : Among all possible representations of the surface, which are equivalent in the sense of Fréchet and which satisfy conditions (a) and (b), there exist some for which the double integral of the sum of the squares of all the first partial derivatives of the functions $x, y, z$ is a minimum:

$$
\iint_{A}\left(x_{u}^{2}+y_{u}^{2}+z_{u}^{2}+x_{v}^{2}+y_{v}^{2}+z_{v}^{2}\right) d u d v \text {. }
$$

This minimum is equal to the Lebesgue area of the surface itself. The minimal representations are all almost conformal and the almost conformal representations are all minimal $[15,17]$.

This problem is considered here as a problem of calculus of variations and is very similar to the problems of Dirichlet and Plateau. Indeed, the proofs of McShane and Morrey contain ideas similar to that used by Lebesgue, Radó, Courant, Tonelli for such variational problems. Using the direct method of calculus of variations, I have proved a theorem of the type of Schwarz's for the representation of polyhedral surfaces, and I have also proved the theorem of Morrey directly [15].

Starting from this result I have, moreover, succeeded in proving the following

THEOREM 4. [23] Each general surface $S$ of finite Lebesgue area admits of a representation upon a circle $A$ such that the Lebesgue area is equal to the classical integral calculated with generalized Jacobians

$$
L(S)=\iint_{A}\left(J_{1}^{2}+J_{2}^{2}+J_{3}^{2}\right)^{1 / 2} d u d v
$$

In such a representation the nondegenerate parts of the surface, that is, in the nomenclature of Rado, the parts that come from the cyclic elements of the middle space, are represented almost conformally. This solves the first representation problem.

Now by means of an observation that was suggested by a previous simpler remark of Youngs, I have proved the following

Theorem 5. [29] Each general surface $S$ of finite Lebesgue area admits of a representation upon a circle $A$ such that

$\left(\mathrm{a}^{*}\right)$ The functions $x, y, z$ have ordinary first partial derivatives a.e. 
in $A$;

(b) the first partial derivatives $x_{u}, x_{v}, \cdots, z_{v}$ are $L^{2}$-integrable functions in $A$;

(c) we have $E=G, F=0$ a.e. in $A$, where $E=x_{u}^{2}+y_{u}^{2}+z_{u}^{2}, G=x_{\text {v }}^{2}$ $+y_{v}^{2}+z_{v}^{2}, F=x_{u} x_{v}+y_{u} y_{v}+z_{u} z_{v}$

(d) the Lebesgue area is given by the classical integral calculated with ordinary Jacobians:

$$
L(S)=\iint_{A}\left(\left[\frac{\partial(y, z)}{\partial(u, v)}\right]^{2}+\left[\frac{\partial(z, x)}{\partial(u, v)}\right]^{2}+\left[\frac{\partial(x, y)}{\partial(u, v)}\right]^{2}\right)^{1 / 2} d u d v .
$$

This theorem solves the second representation problem. Here we have replaced the condition (a) of the theorem of Morrey by the weaker condition $\left(a^{*}\right)$. This fact is related to a remark of Mickle [40]: there exists a surface $S$ of finite Lebesgue area which admits of no representation satisfying the strong condition (a). In this new weaker sense the above theorem can be expressed by saying that each continuous general surface of finite Lebesgue area admits of an almost conformal representation.

These concepts and results are already being applied to the notion of integral upon surfaces in parametric form. I recall here that the notion of integral upon a surface

$$
I_{S}=\iint_{A} F\left(x, y, z, J_{1}, J_{2}, J_{3}\right) d u d v
$$

as a Lebesgue integral was studied in 1932 by McShane $[34,35,37]$ for surfaces $S$ that are given by representations satisfying conditions (a) and (b). More generally Radó [45] studied the same concept in 1944 under the weaker hypotheses that the functions $x, y, z$ have first partial derivatives a.e. in $A$ and that the Lebesgue area is finite and given by the classical integral with ordinary Jacobians.

Recently, in 1946, I [21] introduced the notion of integrals $I_{S}$ as a Weierstrass integral for any continuous surface of finite Lebesgue area given by any continuous representation. I proved that the Weierstrass integral $I_{S}$ is equal to the Lebesgue integral for each representation for which the Lebesgue area is given by the classical integral and, finally, that the necessary conditions [26] and the sufficient [27] conditions determined by McShane and Radó for semicontinuity hold for the class of the most general continuous surfaces. From the above solution of the second representation problem we know now that there always exists at least one representation of the surface $S$ for which the Weierstrass integral $I_{S}$ can be 
calculated as a Lebesgue integral with ordinary Jacobians, that is, as one of the integrals studied by Radó [45].

With all this research in which the efforts of so many mathematicians have been concentrated, perhaps the way is now open for further applications in analysis.

\section{BIBLIOGRAPHY}

\section{A. Books}

RADó, T.

1. Length and area, Amer. Math. Soc. Colloquium Publications, vol. 30, 1948.

2. On the problem of Plateau, Ergebnisse der Mathematik und ihrer Grenzgebiete, vol. $2,1933$.

TONELLI, L.

3. Fondamenti di calcolo delle variazioni, vols. 1 and 2, Bologna, Zanichelli, 1922.

\section{BANACH, S.}

\section{B. ARTICLES}

4. Sur les lignes rectifiables et les surfaces dont l'aire est finie, Fund. Math. vol. 7 (1925) pp. 225-236.

\section{Cesari, L.}

5. Sulle trasformazoni continue, Annali di matematica (4) vol. 21 (1942) pp. 157188.

6. Sulle trasformazioni continue e sull'area delle superficie, Memorie Accademia d'Italia vol. 12 (1941) pp. 1305-1397.

7. Su di un problema di analysis situs dello spazio ordinario, Rendiconti Istituto Lombardo di Scienze e Lettere vol. 75 (1941-1942) pp. 267-291.

8. Caratterizzazione analitica delle superficie continue di area finita secondo Lebesgue, Annali Scuola Normale Superiore di Pisa (2) vol. 10 (1941) pp. 253-294 and vol. 11 (1942) pp. 1-42.

9. Su di un teorema di T. Radó sulle trasformazioni continue, Atti Istituto Veneto di Scienze e Lettere vol. 101 (1941-1942) pp. 377-403.

10. Sui punti di diramazione delle trasformazioni continue e sull'area delle superficie in forma parametrica, Rendiconti di Matematica e delle sue Applicazioni Roma (5) vol. 3 (1942) pp. 37-62.

11. Sui fondamenti geometrici dell'integrale classico per l'area delle superficie in forma parametrica, Memorie Accademia d'Italia vol. 13 (1943) pp. 1323-1481.

12. Sulle quadratura delle superficie in forma parametrica, Bollettino Unione Matematica Italiana (2) Anno IV (1942) pp. 109-117.

13. Una proprietà caratteristica delle trasformazioni a variazione limitata, Bollettino Unione Matematica Italiana (2) Anno IV (1942) pp. 224-235.

14. Sul concetto di trasformazione assolutamente continua, Bollettino Unione Matematica Italiana (2) Anno V (1942) pp. 5-10.

15. Criteri di uguale continuità ed applicazioni alla quadratura delle superficie, Annali Scuola Normale Superiore di Pisa (2) vol. 12 (1943) pp. 61-84.

16. Una uguaglianza fondamentale per l'area delle superficie, Memorie Accademia d'Italia vol. 14 (1944) pp. 891-951.

17. Un complemento alla nota "Criteri di uguale continuitd ed applicazioni alla quadratura delle superficie," Rendiconti Accademia Nazionale Lincei (8) vol. 1 (1946) pp. 292-296. 
18. Rappresentazione quasi conforme delle superficie continue, Rendiconti Accademia Nazionale Lincei (8) vol. 1 (1946) pp. 509-515.

19. Sulla rappresentazione delle superficie continue di area finita secondo Lebesgue, Rendiconti Istituto Lombardo di Scienze e Lettere vol. 79 (1945-1946) pp. $15-45$.

20. Sull'area secondo Lebesgue delle superficie continue, I and II, Rendiconti Accademia Nazionale Lincei (8) vol. 3 (1947) pp. 486-495.

21. La nozione di integrale sopra una superficie in forma parametrica, Annali Scuola Normale Superiore di Pisa (2) vol. 13 (1944) pp. 78-117.

22. Sopra un teorema di approssimazione per le superficie continue in forma parametrica, Rendiconti Accademia Nazionale Lincei (8) vol. 4 (1948) pp. 33-39.

23. Parametrizzazione delle superficie continue di area finita secondo Lebesgue, Annali di Matematica (4) vol. 26 (1947) pp. 301-375.

24. Sulla trasformazione degli integrali doppi, Annali di Matematica (4) vol. 27 (1948) pp. 321-374.

25. Proprietà tangenziali delle superficie continue, Comment. Math. Helv. vol. 22 (1949) pp. 1-16.

26. Condizioni sufficienti per la semicontinuitd degli integrali sopra una superficie in forma parametrica, Annali Scuola Normale Superiore di Pisa (2) vol. 14 (1945) pp. 47-79.

27. Condizioni necessarie per la semicontinuita degli integrali sopra una superficie in forma parametrica, Annali di Matematica (4) vol. 29 (1949) pp. 199-224.

28. Sulle superficie di Fréchet, Rivista di Matematica dell'Università di Parma vol. 1 (1950) pp. 19-44.

29. On the representation of surfaces, Amer. J. Math. vol. 72 (1950) pp. 335-346. Helsel, R. G.

30. A theorem on surface area, Trans. Amer. Math. Soc. vol. 61 (1947) pp. 443-453.

31. Convergence in area and convergence in volume, Duke Math. J. vol. 16(1949) pp. 111-118.

Helsel, R. G., and Mickle, E. J.

32. The Kolmogoroff principle for the Lebesgue area, Bull. Amer. Math. Soc. vol. 54 (1948) pp. 235-238.

LEBESGUE, $\mathrm{H}$.

33. Intégrale, longueur, aire, Annali di Matematica vol. 7. (1902) pp. 231-359.

McShane, E. J.

34. On the semicontinuity of integrals in the calculus of variations, Ann. of Math. vol. 33 (1932) pp. 460-484.

35. Integrals over surfaces in parametric form, Ann. of Math. vol. 34 (1933) pp. 815-838.

36. Parametrizations of saddle surfaces, with applications to the problem of Plateau, Trans. Amer. Math. Soc. vol. 35 (1933) pp. 716-733.

37. Existence theorems for double integral problems of the calculus of variations, Trans. Amer. Math. Soc. vol. 38 (1935) pp. 549-563.

Mickle, E. J.

38. Associated double integral variation problems, Duke Math. J. vol. 9 (1942) pp. 208-227.

39. Metric foundations of continuous transformations, Trans. Amer. Math. Soc. vol. 63 (1948) pp. 368-391.

40. Some examples in surface area theory, Rivista di Matematica dell'Università di Parma vol. 1 (1950). 
Morrey, C. B., JR.

41. A class of representations of manifolds, Amer. J. Math. vol. 55 (1933) pp. 863707 and vol. 56 (1934) pp. 275-293.

42. The toplogy of (path) surfaces, Amer. J. Math. vol. 57 (1935) pp. 17-50.

43. An analytic characterization of surfaces of finite Lebesgue area, Amer. J. Math. vol. 57 (1935) pp. 692-702 and vol. 58 (1936) pp. 313-322.

RaDó, T.

44. The isoperimetric inequality and the Lebesgue definition of surface area, Trans. Amer. Math. Soc. vol. 61 (1947) pp. 530-555.

45. On the semi-continuity of double integrals in the parametric form, Trans. Amer. Math. Soc. vol. 51 (1942) pp. 336-361.

46. (with R. G. Helsel) The Cauchy area of a Frêchet surface, Duke Math. J. vol. 15 (1948) pp. 159-167.

47. (with E. J. Mickle) $A$ new geometrical interpretation of the Lebesgue area of a surface, Duke Math. J. vol. 15 (1948) pp. 169-180.

48. Sur l'aire des surfaces continues, Atti del Congresso Internazionale dei Matematici Bologna vol. 6 (1928) pp. 355-360.

49. (with M. C. Ayer) A note on convergence in length, Bull. Amer. Math. Soc. vol. 54 (1948) pp. 533-539.

50. Two-dimensional concepts of bounded variation and absolute continuity, Duke Math. J. vol. 14 (1947) pp. 587-608.

REICHELDERFER, P. V.

51. On the definition of the essential multiplicity for continuous transformations in the plane, Trans. Amer. Math. Soc. vol. 62 (1947) pp. 284-314.

52. The essential part of a surface, Bull. Amer. Math. Soc. vol. 53 (1947) pp. 845855.

TONELLI, L.

53. Sulla proprietà di minimo della sfera, Rend. Circ. Mat. Palermo vol. 39 (1915) pp. 1-30.

54. Sulla quadratura delle superficie, Rendiconti Accademia Nazionale Lincei (6) vol. 3 (1926) pp. 357-362, 445-450, 633-638, 714-719.

55. Sul problema di Plateau, Rendiconti Accademia Nazionale Lincei (6) vol. 29 (1936) pp. 333-339, 393-398.

56. L'estremo assoluto degli integrali doppi, Annali Scuola Normale Superiore di Pisa (2) vol. 2 (1933) pp. 89-130.

Vitali, G.

57. Sulle funzioni continue, Fund. Math. vol. 8 (1926) pp. 175-188.

Youngs, J. W. T.

58. Curves and surfaces, Amer. Math. Monthly vol. 51 (1944) pp. 1-11.

59. The topological theory of Fréchet surfaces, Ann. of Math. vol. 45 (1944) pp. 753-785.

60. On surfaces of class $K_{1}$, Bull. Amer. Math. Soc. vol. 51 (1945) pp. 669-673.

61. Topological methods in the theory of Lebesgue area, Bull. Amer. Math. Soc. vol. 56 (1950) pp. 17-31.

62. The representation problem for Fréchet surfaces, Memoires of the American Mathematical Society, not yet published.

63. The isoperimetric inequality for closed surfaces, Rivista di Matematica dell'Universita di Parma vol. 1 (1950).

The University of Bologna AND The Ohio State University 